\title{
Efficient Antialiased Edit Propagation for Images and Videos
}

\author{
Li-Qian $\mathrm{Ma}^{\mathrm{a}}$, Kun $\mathrm{Xu}^{\mathrm{a}, *}$ \\ ${ }^{a}$ TNList, Department of Computer Science and Technology, Tsinghua University, Beijing
}

\begin{abstract}
Edit propagation on images/videos has become more and more popular in recent years due to simple and intuitive interaction. It propagates sparse user edits to the whole data following the policy that nearby regions with similar appearances receive similar edits. While it gives a friendly editing mode, it often produces aliasing artifacts on edge pixels. In this paper, we present a simple algorithm to resolve this artifact for edit propagation. The key in our method is a new representation called Antialias Map, in which we represent each antialiased edge pixel by a linear interpolation of neighboring pixels around the edge, and instead of considering the original edge pixels in solving edit propagation, we consider those neighboring pixels. We demonstrate that our work is effective in preserving antialiased edges for edit propagation and could be easily integrated with existing edit propagation methods such as $[1,2]$.
\end{abstract}

Keywords: Antialiasing Recovery, Edit Propagation, Antialias Map

\section{Introduction}

With the development of digital image/video cameras and ${ }_{3}$ online image/video sharing services (e.g. flickr, youtube), it ${ }_{4}$ is much easier for people to access images/videos than before. ${ }_{5}$ The desire to edit the appearance of image/video, such as color, ${ }_{6}$ brightness, tonal values, arises. One way to edit the appearance 7 of images is to first select some regions of interest, and then 8 apply a desired edit operation to those regions. While this is a 9 common solution in commercial softwares such as Photoshop, ${ }_{10}$ selecting those regions of interest, is still a time consuming task, ${ }_{11}$ especially for images with complex textures. Another way is to 12 use edit propagation methods [1, 2, 3]. In these methods, users ${ }_{13}$ only need to specify sparse strokes indicating specific edits (as ${ }_{14}$ shown in Figure 1 (a)), and those edits would be automatically 15 propagate to the whole data following the policy that nearby ${ }_{16}$ regions with similar colors receive similar edits.

17 While edit propagation methods provide a much simpler 18 and more convenient way for editing images/videos, it often ${ }_{19}$ suffers from a visible aliasing artifact. As illustrated in Fig20 ure 1 , in this example, users draw a white stroke on the sky ${ }_{21}$ and a black one on the building, indicating an edit operation 22 that changes color and another edit operation that keeps origi23 nal color, respectively. Figure 1 (b) gives the result generated 24 by a state-of-the-art edit propagation work [1], while it achieves 25 the goal in most parts of the image, however, as shown in the ${ }_{26}$ enlarged image in (b), along the boundary of the building, we ${ }_{27}$ see an undesired, clear edge.

${ }_{28}$ It's not surprising that edit propagation methods would pro29 duce such aliasing artifacts. This is simply because edit propa${ }_{30}$ gation is a per-pixel algorithm and would fail on antialiased pix${ }_{31}$ els. Take Figure 1 as example, in the original image (in Figure 1

${ }^{*}$ Corresponding author
32 (a)), due to its antialiasing nature, the edge pixels exhibit nei${ }_{33}$ ther the color of sky nor the color of the building, but a kind of ${ }_{34}$ blending between the colors of sky and the building. However, 35 under the policy of edit propagation, those antialiased edge pix${ }_{36}$ els are neither similar to the sky pixels nor to the building pixels ${ }_{37}$ due to color differences, this makes appearance of those edge ${ }_{38}$ pixels unchanged after edit propagation, leading to antialiased ${ }_{39}$ edges damaged, as shown in Figure 1 (b). The existence of such 40 artifacts, has largely reduced the fidelity of results and practica${ }_{41}$ bility of edit propagation.

42 To address this issue, in this paper we introduce a novel, ${ }_{43}$ efficient framework to eliminate those aliasing artifacts in edit ${ }_{44}$ propagation. Our work is inspired by a recent work on antialias${ }_{45}$ ing recovery [4], which aims at restoring antialiased edges for 46 a range of image filters. Similar to [4], we assume that for an${ }_{47}$ tialiased edges in images, the value of each pixel could be seen 48 as a linear interpolation from some nearby pixels. Based on this 49 assumption, we introduce a novel representation, the Antialias ${ }_{50}$ Map, which stores the blending weights and relative positions ${ }_{51}$ of nearby interpolating pixels for each edge pixel. While pre52 vious works $[1,2,3]$ directly consider edge pixels in solving ${ }_{53}$ edit propagation, we replace each edge pixel by its interpolat54 ing pixels and use those interpolating pixels in edit propaga${ }_{55}$ tion instead. In turn, the edits of each edge pixel is obtained 56 by an interpolation from those interpolating pixels. As shown 57 in Figure 1 (c), our method successfully preserves the smooth ${ }_{58}$ edge around the boundary of the building after edit propaga59 tion. Furthermore, our method is independent of a specific edit 60 propagation algorithm and could be integrated into any existing ${ }_{61}$ edit propagation methods such as $[1,2,3]$. The results demon62 strate that our method effectively preserves the antialiased s${ }_{63}$ mooth edges without incurring large performance overhead.

64 The rest of the paper is organized as follows: we will first ${ }_{65}$ review some important related works in edit propagation and 


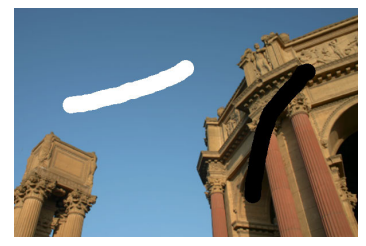

(a) original image

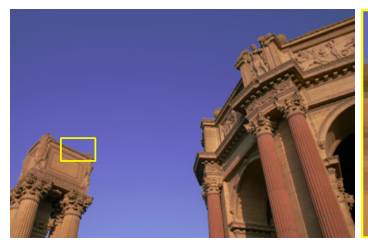

(b) result of Xu et al.

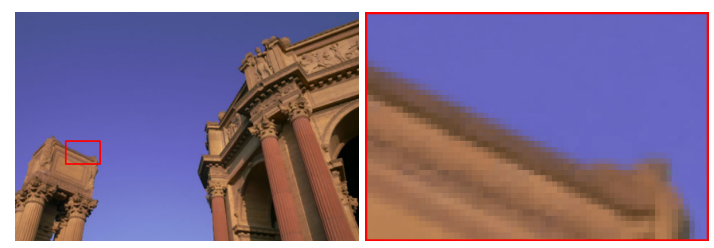

(c) our result

Figure 1: An example of edit propagation. (a) shows the original image and user strokes. (b) and (c) show the propagation results using the method by [1] and our method, respectively. Alias artifacts are visible in (b) along the boundary of the building. Our method successfully eliminate these artifacts, as shown in (c).

66 antialiasing recovery, respectively, in Section 2; the Antialias ${ }_{67}$ Map will be introduced in Section 3; the framework and algo68 rithm details for edit propagation will be explained in Section ${ }_{69} 4$; after that, results and comparisons will be given in Section 5 70 and conclusions will be made in Section 6.

\section{${ }_{71}$ 2. Related Works}

72 In this section we will review some important prior works ${ }_{73}$ in edit propagation and antialiasing recovery, respectively.

\section{2.1. Image/Video Editing}

75 Image/video editing is an increasingly hot topic in comput${ }_{76}$ er graphics in recent years. It could be generally divided into 77 two groups: structural editing $[5,6,7,8,9,10]$ and appear78 ance editing. Appearance editing includes tone editing [11, 12, 79 13, 14, 15, 16], colorization [17, 18, 19, 20], dehazing [21, 22, 80 23, 24, 25], and edge-aware editing [26, 27, 28, 29, 30, 31], 81 etc.. Recently, edit propagation methods [1, 2, 3, 32] allow 82 a simpler interaction mode for appearance editing. In these 83 methods, users specify edits in some sparse locations on im${ }_{84}$ ages/videos, and those edits are automatically propagated to the 85 whole data satisfying the policy that nearby pixels having simi${ }_{86}$ lar appearances receive similar edits. Usually, edit propagation ${ }_{87}$ methods define affinities between pairs of pixels according to 88 their appearance/position distances, and different optimization ${ }_{89}$ schemes are utilized to satisfy the policy. In particular, Pellaci90 ni et al. [32] approximate pixel relations using a sparse graph 91 and reduce edit propagation problem to solving a sparse linear ${ }_{92}$ system. An and Pellacini [3] introduced a more robust algo${ }_{93}$ rithm, which considers all-pairs affinities between pixels, and 94 approximates the huge affinity matrix using a low rank approx95 imation. To accelerate edit propagation, $\mathrm{Xu}$ et al. [1] uses a ${ }_{96} \mathrm{k}$-d tree to organize pixels into hierarchical clusters in a high ${ }_{97}$ dimensional space, instead of propagating on individual pixel${ }_{98} \mathrm{~S}$, they propagate on clusters which largely reduced time and 99 memory cost. Xiao et al. [33] employs a similar hierarchi100 cal structure for acceleration. Li et al. [2] further speeds up 101 edit propagation by formulating it as a function interpolation 102 problem. Bie et al. [34] accelerate edit propagation using stat103 ic clustering and efficient sampling scheme. Besides images 104 and videos, edit propagation could be also used to edit spatially 105 varying bidirectional reflectance distribution functions obtained 106 by $[35,36,37,38]$ and bidirectional texture functions $[39,40]$.
${ }_{107}$ Recently, Farbman et al. [41] proposes to use diffusion distance, 108 instead of Euclidean distance, to define affinities between pix109 els, which better account for the global distribution of pixels.

\section{2.2. Antialiasing Recovery}

111 In computer graphics, many techniques have been proposed 112 to render antialiased images [42, 43], antialiased textures [44, $\left.{ }_{113} 45\right]$ and antialiased shadows [46, 47, 48]. However, only a 114 few works focus on recovering smooth, antialiased edges from 115 aliased 2D images. Some exceptional works include Principle ${ }_{116}$ Component Analysis (PCA) [49, 50] and morphological an117 tialiasing [51]. In particular, morphological antialiasing aims 118 at reducing aliasing artifacts for rendered images entirely us119 ing image based methods. It looks for certain patterns of dis120 continue geometry and replace them using smooth edges esti${ }_{121}$ mated by an antialiased edge model. Image vectorization tech122 niques $[52,53,54]$ convert a bitmap image to a vectorized im${ }_{123}$ age, which could also be used to antialias certain types of im124 ages. Recently, Yang et al. [4] introduced a method for recover125 ing antialiased edges destroyed by a range of non-linear image 126 filters. In this work, an analytic edge model is estimated using ${ }_{127}$ the original image, and is applied to the filtered image to re128 move aliasing artifacts. It works well for many non-linear im129 age filters such as intensity thresholding, tone mapping, color 130 to gray and so on, however, since it requires perfect pixel cor${ }_{131}$ respondence between the original and filtered images, it cannot ${ }_{132}$ handle filters like Gaussian blurring. Besides, it's not clear how ${ }_{133}$ to extend this method to edit propagation.

Compared to the conference paper [55], We have extended 135 our framework to handle interpolation based edit propagation. ${ }_{136}$ This is a significant new contribution compared to [55], since 137 we have demonstrated the proposed Antialias Map is not limit${ }_{138}$ ed to optimization based edit propagation, however, it could al139 so be used for interpolation based edit propagation. This demon140 strates that the proposed Antialias Map is independent with spe${ }_{141}$ cific edit propagation methods and could be potentially com142 bined with any edit propagation methods.

\section{3. Antialias Map}

As mentioned before, since antialiased edges in images are 145 often smooth, we assume the value of an edge pixel could be 146 approximated by a linear interpolation of some nearby pixels. ${ }_{147}$ We present Antialias Map to store those edge pixels. Besides, 
${ }_{148}$ in Antialias Map, for each edge pixel ,we also store the infor149 mation of its neighboring interpolating pixels, including both 150 interpolating weights and relative positions. For videos, we s${ }_{151}$ tore an Antialias Map for every frame. Since our work is built 152 upon the antialiasing recovery work of [4], to make our paper 153 self-contained, before introducing the details of Antialias Map, ${ }_{154}$ we will first explain some necessary backgrounds in [4] in Sec155 tion 3.1.

\section{3.1. Antialiasing Recovery}

157 Images often have smooth, antialiased edges. However, 158 these desired properties will be destroyed by a range of non159 linear image filters, such as intensity thresholding, tone map160 ping, etc.. After applying those image filters, smooth bound${ }_{161}$ aries become zigzag like. Yang et al. [4] proposed a tech162 nique to remove these aliasing artifacts in filtered images. Their 163 method proceeds in several steps:

${ }_{164}$ Edge model. For each pixel $i$ in the original image, they choose 165 the two extremum colors $c_{j}$ and $c_{k}$ ( $j, k$ are corresponding pixel${ }_{166} \mathrm{~S}$ ) in the principle direction of color space from the neighboring ${ }_{167} 8$ pixels (in $3 \times 3$ size neighborhood). The principle direction is 168 determined using an Expectation Maximazation (EM) scheme. ${ }_{169}$ Using extremum colors to reconstruct the color $c_{i}$ of pixel $i$, the 170 interpolation weights $\alpha_{i j}, \alpha_{i k}$ could be determined by minimiz171 ing:

$$
d_{i}=\left\|\left(\alpha_{i j} c_{j}+\alpha_{i k} c_{k}\right)-c_{i}\right\|
$$

172 where it satisfies $\alpha_{i j}+\alpha_{i k}=1$.

${ }_{173}$ Probability of lying on edges. After that, they estimate the 174 probability of each pixel that it lies on an edge. For each pixel $175 i$, They define an edge strength $e_{i}$, which is the product of the ${ }_{176}$ Sobel edge detector at both the original image and the filtered 177 image. The probability value of a pixel lying on an edge is 178 defined as:

$$
\beta_{i}=G\left(d_{i}, \sigma_{d}\right)\left(1-G\left(e_{i}, \sigma_{e}\right)\right)
$$

179 where $G(d, \sigma)$ is a $1 \mathrm{D}$ Gaussian defined as $\exp \left(-d^{2} / \sigma^{2}\right), d_{i}$ is ${ }_{180}$ the residual distance defined in Equation $1, \sigma_{d}$ and $\sigma_{e}$ are two ${ }_{181}$ controllable parameters. $\beta_{i}$ is set as zero if $e_{i}>3 \sigma_{e}$.

${ }_{182}$ Recovery the filtered image. Denote $f_{i}$ is the color value of ${ }_{183}$ pixel $i$ on the filtered image. The recovered color value $r_{i}$ could ${ }_{184}$ be obtained by solving the linear system below:

$$
r_{i}=\beta_{i}\left(\alpha_{i j} r_{j}+\alpha_{i k} r_{k}\right)+\left(1-\beta_{i}\right) f_{i}
$$

${ }_{185}$ This is a large sparse linear system and could be solved effi186 ciently by a few iterations using the Jacobi method.

\section{3.2. Compute Antialias Map}

${ }_{188}$ As discussed in Section 3.1, in [4], the value of each an189 tialiased edge pixel is approximated by a blending of 2 near190 by pixels in the $3 \times 3$ neighborhood. Results are progressively 191 refined by iterations of Equation 3. Instead of using a $3 \times 3$ 192 neighborhood, Antialias Map approximates the value of each 193 pixel by a blending of pixels from a larger neighborhood:

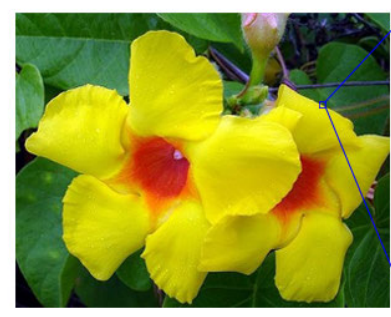

(a) source image

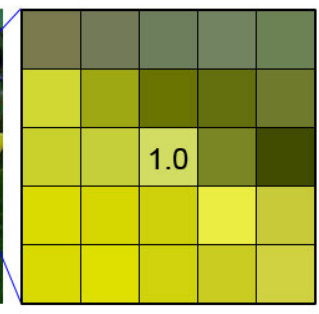

(b) initial value

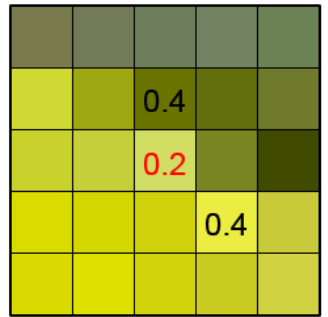

(c) 1 iteration

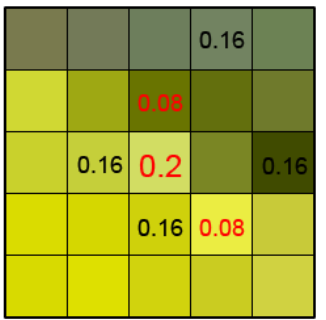

(d) 2 iterations
Figure 2: Antialias Map construction. (a) is the source image; (b),(c) and (d) give the Antialias Map of a certain pixel after 0,1,2 iterations, respectively. Divisible pixels are colored black, while indivisible pixels are colored red.

$$
c_{i} \approx \sum_{j} w_{i j} c_{j}
$$

194 where $j$ is the interpolating pixel in the neighborhood of $i$, and $195 w_{i j}$ is the interpolating weight from pixel $i$ to $j$, and satisfies ${ }_{196} \sum_{j} w_{i j}=1$. Note that $w_{i j}$ does not necessarily equal to $w_{j i}$. Al197 So note that Equation 4 is not an optimization target, and the 198 interpolating weights are not solved from Equation 4. Instead, 199 the interpolating weights are computed through an iteractive ap200 proach, which will be explained in detail below.

201 Antialias Map has two advantages over the edge model pro202 posed in [4]. First, since it uses a larger neighborhood to ap${ }_{203}$ proximate an antialiased pixel, it leads to a more accurate ap204 proximation; Secondly, the Antialias Map only depends on the 205 structure of original image itself, it could be computed and s206 tored before edit propagation, so it avoids the cost of iterations 207 at run-time edit propagation stage. Antialias Map stores all in208 terpolating weights $w_{i j}$, and it is sparse since it only considers 209 those edge pixels (e.g. whose edge strength $\beta_{i}$ is non-zero) and 210 it only stores non-zero weights. Specifically, we store a set of 211 triples $\left(\Delta x_{i j}, \Delta y_{i j}, w_{i j}\right)$ for each edge pixel $i$. Here $j$ is its inter${ }_{212}$ polating pixel, $\Delta x_{i j}, \Delta y_{i j}$ and $w_{i j}$ are the $\mathrm{x}, \mathrm{y}$ position offset and ${ }_{213}$ interpolating weight from $i$ to $j$, respectively. In the follow214 ing parts, we will explain how to compute the Antialias Map in 215 detail.

Initialization. In this step, we first use [4] to obtain the two ${ }_{217}$ extremum neighbors $j, k$, the blending factors $\alpha_{i j}, \alpha_{i k}$ and the ${ }_{218}$ edge probability $\beta_{i}$ for each pixel $i$. We have already explained ${ }_{219}$ how to compute those values in Section 3.1. Care must be taken 220 when computing the edge probability $\beta_{i}$. In [4], it defines edge ${ }_{221}$ strength of each pixel as the product of Sobel edge detector on 


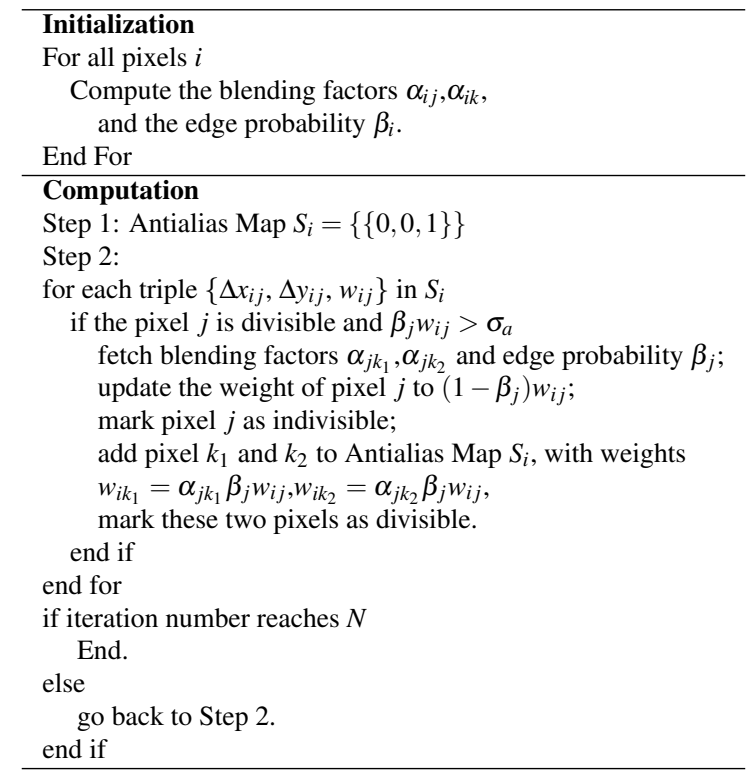

Table 1: Pseudocode for Antialias Map Construction.

222 both original and filtered images, which means it requires to ob223 tain the aliased filtered image before antialiasing recovery. We 224 observe that in edit propagation, the appearances are changed 225 smoothly, so that the propagated result images have roughly 226 the same structure as the original images. To avoid the cost to 227 generate an aliased edit propagation result, we make a modifi228 cation, instead, we define the edge strength as the Sobel edge 229 detector only on the original image. Once the edge strength is 230 computed, we use Equation 2 to compute edge probability $\beta_{i}$. ${ }_{231}$ Note that only the pixels with non-zero $\beta_{i}$ are considered as an${ }_{232}$ tialiased edge pixels and stored in Antialias Map. The pixels ${ }_{233}$ with zero value of $\beta_{i}$ are considered as non-edge pixels.

${ }_{234}$ Constructions. Similar to [4], we construct Antialias Map 235 by a few iterations. However, they obtain the final antialiased 236 results through iterations, but we obtain Antilias Map through ${ }_{237}$ iterations, which could be precomputed and stored before edit ${ }_{238}$ propagation. For each antialiased edge pixel $i$, the Antialias ${ }_{239}$ Map starts with a set containing only one triple:

$$
S_{i}=\{\{0,0,1\}\}
$$

${ }_{240}$ This means that the value of the pixel $i$ could be seen as the 241 value of itself multipled by weight 1.0 , which is definitely true. ${ }_{242}$ We also illustrate this iteration process in Figure 2. As shown 243 in Figure 2 (b), now the Antialias Map only contains itself with 244 weight 1.0. And this pixel is marked as divisible, which is paint245 ed using black color in Figure 2.

${ }_{246}$ At each iteration, we expand each divisible pixel (e.g. $j$ ) 247 into 3 pixels. These 3 pixels are the two neighboring extremum ${ }_{248}$ pixels (e.g., $k_{1}$ and $k_{2}$ ) and itself (e.g. $j$ ), whose corresponding 249 weights are defined in Equation 3. Specially, the weight of $j$ 250 is replaced by $\left(1-\beta_{j}\right) w_{i j}$ and $j$ is marked as indivisible; the 251 two newly added extremum pixels are marked as divisible, and ${ }_{252}$ their weights are set as $w_{i k_{1}}=\alpha_{j k_{1}} \beta_{j} w_{i j}$ and $w_{i k_{2}}=\alpha_{j k_{2}} \beta_{j} w_{i j}$, ${ }_{253}$ respectively. At the next iterations, we recursively find the di-

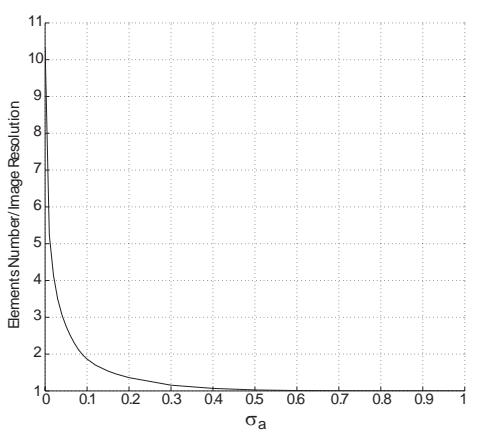

Figure 3: the size of Antialias Map to the size of image as a function of threshold $\sigma_{a}$. This curve is generated from a $240 \mathrm{~K}$ photographed image (the image in Figure 1) and using maximum iteration number $N=4$.

254 visible pixels and expand them to new pixels.

255 Let's also take Figure 2 as example and explain this process 256 in detail. For simplicity, we assume that for all the pixels, the ${ }_{257}$ edge probability $\beta$ is 0.8 and the blending factor $\alpha$ is 0.5 . After 258 the first iteration, the center pixel is expanded to 3 pixels, so 259 that the Antialias Map grows to contain 3 triples (as shown in 260 Figure 2 (c) ):

$$
S_{i}=\{\{0,0,0.2\},\{0,-1,0.4\},\{1,1,0.4\}\}
$$

After the second iterations, similarly, the newly added 2 pix262 els in first iteration are both expanded to 3 pixels, so that the ${ }_{263}$ Antialias Map grows to contain 7 triples (as shown in Figure 2 264 (d) ):

$$
S_{i}=\{\{0,0,0.2\},\{0,-1,0.08\},\{1,1,0.08\},
$$

$$
\{-1,0,0.16\},\{1,-2,0.16\},\{0,1,0.16\},\{2,0,0.16\}\}
$$

Notice that at all iterations, the sum of weights equals to 266 one. From an algebraic aspect, Antialias Map could also be 267 treated as expanding Equation 3 to multiple variables. The 268 triples in Antialias Map will extend to $(2 N+1) \times(2 N+1)$ 269 neighborhood after $\mathrm{n}$ iterations.

${ }_{270}$ Stop Criterion. The size of the Antialias Map grows as we 271 iterate. We define 2 criterions to stop the recursive iteration:

- When iteration number reaches a predefined number $N$;

- When the result product(product of the interpolation weight of a divisible pixel $w_{i j}$ and its edge probability $\beta_{j}$ ) is smaller than a predefined threshold $\sigma_{a}$.

The pseudocode of Antialias Map construction is given in Ta277 ble 1 . We have also tested how two parameters influence the 278 performance of our algorithm. Figure 3 illustrates the size of 279 Antialias Map to the size of image as a function of weight 280 threshold $\sigma_{a}$. Setting $\sigma_{a}=0$ means the iteration stops only 281 when it reaches the largest iteration number $N$, while setting ${ }_{282} \sigma_{a}=1$ means no iteration. As shown in Figure 3, when in${ }_{283}$ creasing $\sigma_{a}$ from 0 to 1 , the size of Antialias Map decreases 284 rapidly. 


\section{4. Improved Framework of Edit Propagation}

286 In this section we will discuss how to use Antialias Map in 287 the pipeline of edit propagation to remove the aliasing artifacts. 288 In edit propagation, users specify edits in some sparse locations 289 on images/videos, and those edits are automatically propagated 290 to the whole data satisfying the policy that nearby pixels having 291 similar appearances receive similar edits. Usually, they define 292 a feature vector for each pixel, usually a 5-dimensional vector ${ }_{293}$, which combines color (e.g. 3D), pixel position (e.g. 2D). For 294 videos, another dimensional is added to account for time. The 295 affinity between every two pixels are defined by the Euclidean 296 distance between their feature vectors, which is then used to 297 guide the propagation. Commonly, edit propagation methods 298 could be divided into two groups, depending on which scheme 299 is used to formulate the problem: optimization based [1, 3] and 300 interpolation based [2]. We show that Antialias Map could be 301 used in both groups for antialias recovery.

\section{4.1. Optimization based Edit Propagation}

${ }_{30 з}$ Backgrounds. As mentioned above, the affinity value between 304 two pixels $i, j$ is usually defined as:

$$
z_{i j}=\exp \left(-\left(\mathbf{f}_{i}-\mathbf{f}_{j}\right)^{2}\right)
$$

305 where $\mathbf{f}_{i}$ is the feature vector of pixel $i$, which is defined as a 5D 306 vector for images:

$$
\mathbf{f}_{i}=\left(c_{i} / \sigma_{c}, p_{i} / \sigma_{p}\right)
$$

307 where $c_{i}, p_{i}$ is the color in LAB color space and the pixel po308 sition of pixel $i$, respectively. $\sigma_{c}$ and $\sigma_{p}$ are two parameters to 309 control the relative propagating distance.

${ }_{310}$ In [3], edit propagation is formulated as an optimization ${ }_{311}$ problem. Solving propagated edits $e$ is deduced to minimize 312 the energy function below:

$$
\sum_{i, j} b_{j} z_{i j}\left(e_{i}-g_{j}\right)^{2}+\lambda \sum_{i, j} z_{i j}\left(e_{i}-e_{j}\right)^{2}
$$

313 where $i, j$ enumerates all pixels; $b_{j}$ is 1 when pixel $j$ is covered 314 by stroke and is 0 elsewhere; $g_{j}$ is the user specified edit at pix${ }_{315} \mathrm{el} j ; e_{i}$ is the propagated edit at pixel $i$ that we want to solve. ${ }_{316}$ The first term accounts for how it satisfies user input while the 317 second term accounts for the edit propagation policy that simi${ }_{318}$ lar pixels receive similar edits. $\lambda$ is used to control the relative 319 weight between the two terms and is usually set to $\sum_{j} b_{j} / \sum_{j} 1$ 320 to make the two terms have roughly the same contributions.

${ }_{321}$ Since the energy function in Equation 10 is quadratic, min322 imizing it is equivalent to solving a linear system defined by ${ }_{323}$ a large affinity matrix. Therefore, they used low rank colum$324 \mathrm{n}$ sampling to approximate the affinity matrix and further pro325 posed an approximated algorithm to fast find a solution. To 326 accelerate edit propagation and extend it to videos, $\mathrm{Xu}$ et al. [1] ${ }_{327}$ proposed to use $\mathrm{k}-\mathrm{d}$ tree to organize pixels into hierarchical 328 clusters. Instead of propagating on pixels, they propagate on 329 clusters, whose number is much smaller than the number of 330 pixels, thus acceleration is achieved. Finally, edits of individual
331 pixels are obtained by multi-linear interpolation from clusters. з32 They also adopted an optimization based method to solve for ${ }_{333}$ edit propagation.

${ }_{334}$ Modified Formulation. As illustrated in the teaser image, tra335 ditional edit propagation produces artifacts on object bound${ }_{336}$ aries. This artifact could be easily explained. Assume a very ${ }_{337}$ simple image composed of 2 region, one red region and anoth${ }_{338} \mathrm{er}$ blue region. The edge pixels along the boundary of the two ззя regions would appear yellow due to antialiasing. Suppose user 340 specifies some edits on the red region, it is also desired to prop341 agate the edits to the edge pixels with some weight according to 342 antialiasing opacity. However, since the edge pixels appearance 343 yellow, it exhibits a large difference to pixels in the red region, 344 hence would not receive any propagated edits.

To address this issue, we use Antialias Map, in which, the yellow edge pixels would be represented by a linear blending of some red and blue neighboring pixels. Instead of propagating to the edge pixels, we propagate to the neighboring interpolating pixels, and obtain the edit of edge pixel by blending the edits from the interpolating pixels. Mathematically, we modify the formulation in Equation 10 to:

$$
\sum_{i, j} b_{j} \gamma_{i} \gamma_{j} z_{i j}\left(e_{i}^{\prime}-g_{j}^{\prime}\right)^{2}+\lambda \sum_{i, j} \gamma_{i} \gamma_{j} z_{i j}\left(e_{i}^{\prime}-e_{j}^{\prime}\right)^{2}
$$

345 where $i, j$ enumerates all interpolating pixels; $\gamma_{i}$ considers the 346 multiplicity of pixel $i$ serving as interpolating pixels, which is ${ }_{347}$ defined as $\gamma_{i}=\sum_{k} w_{k i} ; g_{j}^{\prime}$ is defined as $g_{j}^{\prime}=\sum_{k} w_{k j} g_{j} / \sum_{k} w_{k j}$.

348 The modified energy function has the same form as the orig349 inal energy function in Equation 10, so that it could be solved 350 in the same way using either low rank column sampling [3] or $351 \mathrm{k}-\mathrm{d}$ tree clustering [1].

352 Interpolation. After solving for the edits $e^{\prime}$ on the interpolating ${ }_{353}$ pixels in Equation 11, it is easy to obtain the edits on the edge ${ }_{354}$ pixels through interpolation:

$$
e_{i}=\sum_{j} w_{i j} e_{j}^{\prime}
$$

\section{4.2. Interpolation based Edit Propagation}

${ }_{356}$ Backgrounds. While most works adopt an optimization based 357 method to solve edit propagation, Li et al. [2] proposed a d358 ifferent approach. They observe that the edits span in the high 359 dimensional feature space form a smooth function, which could 360 be approximated well by function interpolations. Therefore, 361 they use sum of RBFs (Radial Basis Function) to approximate 362 edits:

$$
e_{i} \approx \sum_{m} a_{m} G\left(\left\|\mathbf{f}_{i}-\mathbf{f}_{m}\right\|\right)
$$

363 where $m$ iterates over all RBFs; $G$ is RBF Gaussian function; ${ }_{364} a_{m}, \mathbf{f}_{m}$ is the $m$-th RBF coefficient and center, respectively. The 365 centers of RBFs are randomly selected from the pixels covered 366 by user stroke. The coefficients of RBFs are solved by mini367 mizing the sum of differences on user specified edits:

$$
\sum_{j}\left(g_{j}-\sum_{m} a_{m} G\left(\left\|\mathbf{f}_{j}-\mathbf{f}_{m}\right\|\right)\right)^{2}
$$


368 where $j$ iterates over all pixels covered by user strokes. To re369 strict the coefficients to be non-negative, they use a non-negative 370 least square solver.

371 Modified Formulation. The above formulation would also 372 produce aliasing artifacts on object boundaries. To remove the 373 artifacts using Antialias Map, similarly, we build the smooth 374 function over the interpolating pixels, instead of the original 375 pixels. Equation 14 is modified to:

$$
\sum_{j} \gamma_{j}\left(g_{j}^{\prime}-\sum_{m} a_{m} G\left(\left\|\mathbf{f}_{j}-\mathbf{f}_{m}\right\|\right)\right)^{2}
$$

376 where $j$ iterates over all interpolating pixels that have contri377 butions to user stroke pixels; $\gamma_{j}$ considers the multiplicity of ${ }_{378}$ pixel $j$ serving as interpolating pixels, which is defined as $\gamma_{j}=$ 379 $\sum_{k} w_{k j} ; g_{j}^{\prime}$ is defined as $g_{j}^{\prime}=\sum_{k} w_{k j} g_{j} / \sum_{k} w_{k j}$, where $k$ is iter380 ating over user stroke pixels.

381 After solving for the RBF coefficients, we use Equation 13 382 to obtain the edits on interpolating pixels. Lastly, we use Equa${ }_{383}$ tion 12 to obtain the edits on the edge pixels.

\section{${ }_{384}$ 5. Comparisons and Results}

\section{5.1. Comparisons}

${ }_{386}$ Comparison of weight threshold $\sigma_{a}$. In Figure 4, we have 387 compared edit propagation results generated by $\mathrm{Xu}$ et al. [1] 388 and by our method with different weight threshold $\sigma_{a}$. From 389 the results, we can see artifacts using the method by $\mathrm{Xu}$ et al, 390 where the pixels along the boundary of the toy undesirably ap391 pear green. Using a large value of $\sigma_{a}$ (e.g. $\left.\sigma_{a}=0.8,0.4\right)$ still 392 produce these artifacts. But using a relatively small value of $\sigma_{a}$ ${ }_{39 з}$ (e.g. $\sigma_{a}=0.1,0.0$ ) fully removes the artifacts.

${ }_{394}$ Comparison of maximum iteration number $N$. In Figure 5, 395 we have compared edit propagation results generated by $\mathrm{Xu}$ 396 et al. [1] and by our method with different maximum iteration 397 number $N$. From the results, we can see that using a relatively 398 large value of $N$ (e.g. $N=4,8$ ) could produce smooth transi399 tions along boundaries.

\section{5.2. Results}

401 All these results and performance are obtained using a con402 sumer level PC with a 3.0GHz Intel Core2Duo CPU and 4GB ${ }_{403}$ RAM. As demonstrated in the comparisons, setting $\sigma_{a}=0.1$ 404 and $N=4$ already leads to very good results. So in our imple405 mentation, we fix $\sigma_{a}=0.1$ and $N=4$. These two parameters ${ }_{406}$ could still be adjusted for better performance or accuracy. In 407 our experiment, for a single image, the total size of Antialias ${ }_{408}$ Map (e.g. the total number of triples) is usually about $1.5-2.0$ 409 times of the image resolution. So that it only needs small extra 410 space to store the Antialias Map.

${ }_{411}$ In Figure 6, we give 2 image results generated by the k-d ${ }_{412}$ tree approach [1] and by our method. In Figure 7, we give 2 ${ }_{413}$ image results generated by the RBF interpolation approach [2] ${ }_{414}$ and by our method. In Figure 8, we give 1 image result gener415 ated by AppProp [3] and by our method. In Figure 9, we com${ }_{416}$ pare a video example using the k-d tree approach [2] and using ${ }_{417}$ our method, respectively. In all these examples, after applying
418 our methods, the aliasing artifacts along the object boundaries 419 are successfully removed. The performance value is reported in 420 Table 2. Note that the time cost reported for the video example ${ }_{421}$ in Table 2 is the time for processing the whole video (all the 422 frames). It could be substantially accelerated for fast preview${ }_{423}$ ing purposes, when users desire to see a single (or a few) frames 424 of the video, and only the pixels on the previewing frames need 425 to be propagated.

\section{${ }_{426}$ 6. Conclusion}

In this paper we have presented a novel, efficient approach 428 to remove aliasing artifacts in edit propagation. we introduced 429 a novel representation, the Antialias Map, to store the blending 430 weights and relative positions of nearby interpolating pixels for 431 each edge pixel. While previous works $[1,2,3]$ directly consid${ }_{432}$ er edge pixels in edit propagation process, instead, we replace ${ }_{433}$ each edge pixel by its interpolating pixels and consider those 434 interpolating pixels in edit propagation process. Our method is 435 independent of a specific edit propagation algorithm and could 436 be integrated into any existing edit propagation methods such as $437[1,2,3]$. The results demonstrates that our method effectively 438 and efficiently restores the antialiased smooth edges.

${ }_{439}$ There are some works that we would like to address in the 440 future. First, we currently deal with videos frame by frame, and ${ }_{441}$ for each frame we use a 2D Antialias Map. We would like to 442 explore methods to extend Antialias Map to a 3D representa443 tion so that it could also handle motion blurs in the temporal di444 mension; Secondly, we would like to investigate how Antialias 445 Map could be used for other image related applications, such as 446 image compositing [56, 57, 58, 59] and non-photorealistic ren${ }_{447}$ dering [60], since it is also desired to preserve antialiased edges 448 when compositing new images.

\section{7. Acknowledgements}

450 We thank all the reviewers for their valuable comments and 451 insightful suggestions. This work was supported by the Na452 tional Basic Research Project of China ( Project Number $4532011 \mathrm{CB} 302205$ ), the Natural Science Foundation of China ( ${ }_{454}$ Project Number 61120106007 and 61170153 ).

\section{References}

[1] Xu K, Li Y, Ju T, Hu SM, Liu TQ. Efficient affinity-based edit propagation using k-d tree. ACM Transactions on Graphics 2009;28(5):118:1118:6.

[2] Li Y, Ju T, Hu SM. Instant propagation of sparse edits on images and videos. Computer Graphics Forum 2010;29(7):2049-2054.

[3] An X, Pellacini F. Appprop: all-pairs appearance-space edit propagation. ACM Transactions on Graphics 2008;27(3):40:1-40:9.

[4] Yang L, Sander PV, Lawrence J, Hoppe H. Antialiasing recovery. ACM Transactions on Graphics 2011;30(3):22:1-22:9.

[5] Pérez P, Gangnet M, Blake A. Poisson image editing. ACM Transactions on Graphics 2003;22:313-318.

[6] Cheng MM, Zhang FL, Mitra NJ, Huang X, Hu SM. Repfinder: finding approximately repeated scene elements for image editing. ACM Transactions on Graphics 2010;29:83:1-83:8.

[7] Huang H, Zhang L, Zhang HC. Repsnapping: Efficient image cutout for repeated scene elements. Computer Graphics Forum 2011;30:2059-2066. 


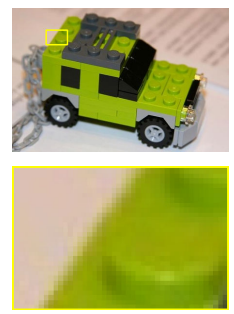

(a) source image

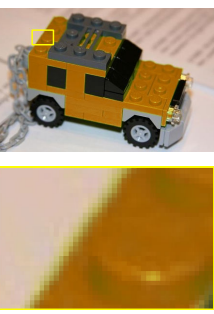

(b) Xu et al.

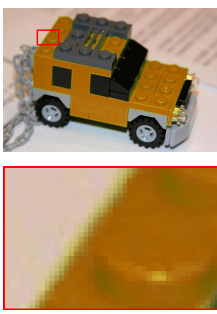

(c) $\sigma_{a}=0.8$

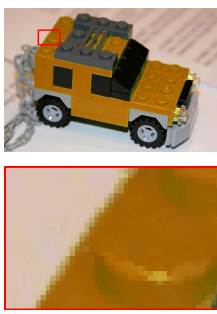

(d) $\sigma_{a}=0.4$

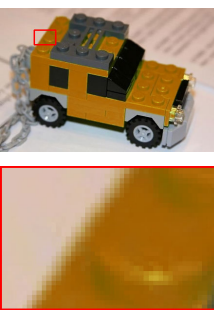

(e) $\sigma_{a}=0.1$

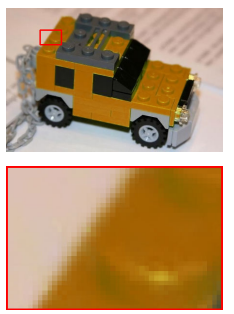

(f) $\sigma_{a}=0.0$

Figure 4: Comparison of edit propagation results generated by Xu et al. [1] and by our method with different weight threshold $\sigma_{a}$. (a) is the source image $O .(b)$ is the edit propagation result of [1], artifacts can be found along the boundaries. (c)-( $f$ ) are results using our algorithm with $\sigma_{a}=0.8,0.4,0.1,0.0$.
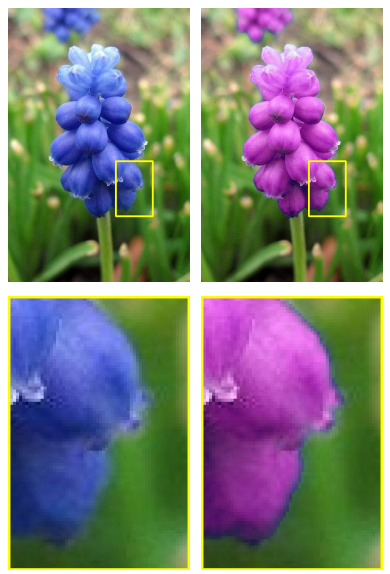

(a) source

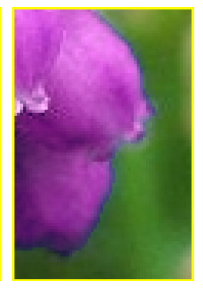

(b) Xu et al.
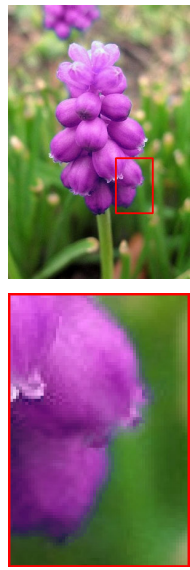

(c) $N=1$
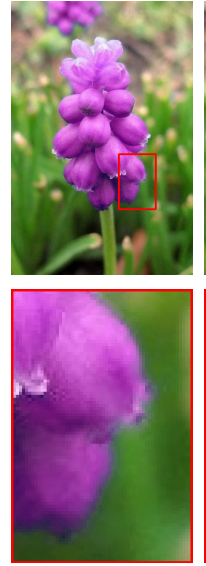

(d) $N=2$
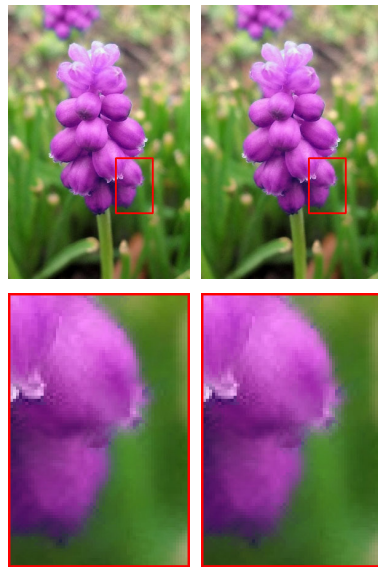

(e) $N=4$

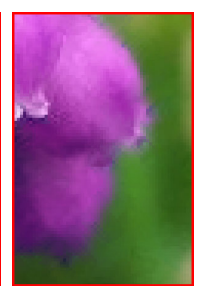

(f) $N=8$

Figure 5: Comparison of edit propagation results generated by Xu et al. [1] and by our method with different maximum iteration number $N .(a)$ is the source image. $(b)$ is the edit propagation result generated by Xu et al.. Notice that artifacts can be found along the boundaries. (c)-(f) are results using our method with $N=1,2,4,8$, respectively.

[8] Zhang Y, Tong R. Environment-sensitive cloning in images. The Visual Computer 2011;27(6-8):739-48.

9] Seol Y, Seo J, Kim PH, Lewis JP, Noh J. Weighted pose space editing for 502 facial animation. The Visual Computer 2012;28(3):319-27.

0] Yang F, Li B. Unsupervised learning of spatial structures shared among images. The Visual Computer 2012;28(2):175-80.

1] Reinhard E, Ward G, Pattanaik S, Debevec P. High dynamic range imaging: Acquisition, Display, and Image-Based Lighting. 2005.

] Gooch AA, Olsen SC, Tumblin J, Gooch B. Color2gray: saliencepreserving color removal. ACM Transactions on Graphics 2005;24:634639. local adjustment of tonal values. ACM Transactions on Graphics 512 2006;25(3):646-653.

[14] Huang H, Xiao X. Example-based contrast enhancement by gradient 5 mapping. The Visual Computer 2010;26:731-738.

15] Pajak D, Čadík M, Aydın TO, Okabe M, Myszkowski K, Seidel HP. Con- 5 trast prescription for multiscale image editing. The Visual Computer 2010;26:739-748.

6] Wu J, Shen X, Liu L. Interactive two-scale color-to-gray. The Visual Computer 2012;28(6-8):723-31.

Welsh T, Ashikhmin M, Mueller K. Transferring color to greyscale images. ACM Transactions on Graphics 2002;21(3):277-280.

Levin A, Lischinski D, Weiss Y. Colorization using optimization. ACM Transactions on Graphics 2004;23(3):689-694.

Yatziv L, Sapiro G. Fast image and video colorization using chrominance blending. IEEE Transactions on Image Processing 2006;15(5):11201129
500 [20] Xiao X, Ma L. Color transfer in correlated color space. In: Proceedings of the 2006 ACM international conference on Virtual reality continuum and its applications. VRCIA '06; 2006, p. 305-309.

503 [21] Fattal R. Single image dehazing. ACM Transactions on Graphics 2008;27:72:1-72:9.

[22] He K, Sun J, Tang X. Single image haze removal using dark channel prior. IEEE Transactions on Pattern Analysis and Machine Intelligence 2010;99:2341-2353.

8 [23] Ding M, Tong R. Efficient dark channel based image dehazing using quadtrees. Science China Information Science 2012;

[24] Zhang J, Li L, Zhang Y, Yang G, Cao X, Sun J. Video dehazing with spatial and temporal coherence. The Visual Computer 2011;27(6-8):74957.

[25] Xiao C, Gan J. Fast image dehazing using guided joint bilateral filter. The Visual Computer 2012;28(6-8):713-21.

515 [26] Chen J, Paris S, Durand F. Real-time edge-aware image processing with the bilateral grid. ACM Transactions on Graphics 2007;26(3).

7 [27] Li Y, Adelson E, Agarwala A. Scribbleboost: Adding classification to edge-aware interpolation of local image and video adjustments. Computer Graphics Forum 2008;27(4):1255-1264.

520 [28] Fattal R, Carroll R, Agrawala M. Edge-based image coarsening. ACM Transactions on Graphics 2009;29(1):6:1-6:11.

522 [29] Fattal R. Edge-avoiding wavelets and their applications. ACM Transactions on Graphics 2009;28(3):22:1-22:10.

524 [30] Criminisi A, Sharp T, Rother C, P'erez P. Geodesic image and video editing. ACM Transactions on Graphics 2010;29:134:1-134:15.

526 [31] Xie ZF, Lau RWH, Gui Y, Chen MG, Ma LZ. A gradient-domain-based edge-preserving sharpen filter. The Visual Computer 2012; 


\begin{tabular}{|c|c|c|c|c|c|c|c|c|c|}
\hline \multicolumn{2}{|c|}{ Data } & $\begin{array}{c}\text { toy } \\
\text { (Fig. 4) }\end{array}$ & $\begin{array}{c}\text { flower } \\
\text { (Fig. 5) }\end{array}$ & $\begin{array}{c}\text { cake } \\
\text { (Fig. 7) }\end{array}$ & $\begin{array}{c}\text { dog } \\
\text { (Fig. 7) }\end{array}$ & $\begin{array}{l}\text { branch } \\
\text { (Fig. 6) }\end{array}$ & $\begin{array}{c}\text { parrot } \\
\text { (Fig. 6) }\end{array}$ & $\begin{array}{c}\text { sky } \\
\text { (Fig. 1) }\end{array}$ & $\begin{array}{c}\text { bird } \\
\text { (Fig. 8) }\end{array}$ \\
\hline \multirow{2}{*}{\multicolumn{2}{|c|}{$\begin{array}{c}\text { Type } \\
\text { Resolution }\end{array}$}} & image & image & image & image & image & image & image & video \\
\hline & & $120 \mathrm{~K}$ & $120 \mathrm{~K}$ & $120 \mathrm{~K}$ & $120 \mathrm{~K}$ & $150 \mathrm{~K}$ & $150 \mathrm{~K}$ & $240 \mathrm{~K}$ & $30 \mathrm{M}$ \\
\hline \multicolumn{2}{|c|}{ Frame Num } & - & - & - & - & - & - & - & 400 \\
\hline \multirow{2}{*}{ K-d tree } & time & $22 \mathrm{~ms}$ & $23 \mathrm{~ms}$ & $17 \mathrm{~ms}$ & $25 \mathrm{~ms}$ & $28 \mathrm{~ms}$ & $24 \mathrm{~ms}$ & $41 \mathrm{~ms}$ & $8 \mathrm{~s}$ \\
\hline & memory & $8 \mathrm{MB}$ & $8 \mathrm{MB}$ & $8 \mathrm{MB}$ & $8 \mathrm{MB}$ & $8 \mathrm{MB}$ & $8 \mathrm{MB}$ & $8 \mathrm{MB}$ & $22 \mathrm{MB}$ \\
\hline \multirow{2}{*}{$\begin{array}{c}\text { Improved } \\
\text { k-d tree }\end{array}$} & time & $40 \mathrm{~ms}$ & $42 \mathrm{~ms}$ & $32 \mathrm{~ms}$ & $45 \mathrm{~ms}$ & $45 \mathrm{~ms}$ & $47 \mathrm{~ms}$ & $79 \mathrm{~ms}$ & $13 \mathrm{~s}$ \\
\hline & memory & $9 \mathrm{MB}$ & $9 \mathrm{MB}$ & $9 \mathrm{MB}$ & $9 \mathrm{MB}$ & $9 \mathrm{MB}$ & $9 \mathrm{MB}$ & $9 \mathrm{MB}$ & $24 \mathrm{MB}$ \\
\hline \multirow{2}{*}{ RBF } & time & $16 \mathrm{~ms}$ & $17 \mathrm{~ms}$ & $13 \mathrm{~ms}$ & $20 \mathrm{~ms}$ & $21 \mathrm{~ms}$ & $19 \mathrm{~ms}$ & $26 \mathrm{~ms}$ & $4 \mathrm{~s}$ \\
\hline & memory & $1 \mathrm{MB}$ & $1 \mathrm{MB}$ & $1 \mathrm{MB}$ & $1 \mathrm{MB}$ & $1 \mathrm{MB}$ & $1 \mathrm{MB}$ & $1 \mathrm{MB}$ & $1 \mathrm{MB}$ \\
\hline \multirow{2}{*}{$\begin{array}{c}\text { Improved } \\
\text { RBF }\end{array}$} & time & $32 \mathrm{~ms}$ & $30 \mathrm{~ms}$ & $25 \mathrm{~ms}$ & $38 \mathrm{~ms}$ & $32 \mathrm{~ms}$ & $36 \mathrm{~ms}$ & $51 \mathrm{~ms}$ & $8 \mathrm{~s}$ \\
\hline & memory & $1 \mathrm{MB}$ & $1 \mathrm{MB}$ & $1 \mathrm{MB}$ & $1 \mathrm{MB}$ & $1 \mathrm{MB}$ & $1 \mathrm{MB}$ & $1 \mathrm{MB}$ & $1 \mathrm{MB}$ \\
\hline
\end{tabular}

Table 2: Performance comparison between the $k$-d tree method [1], our method combined with the k-d tree approach, RBF method [2] and our method combined with the RBF method. Both running time and memory cost are reported.

original image

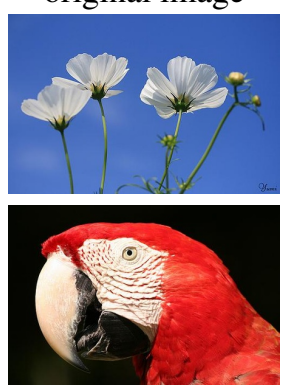

result of $\mathrm{Xu}$ et al.
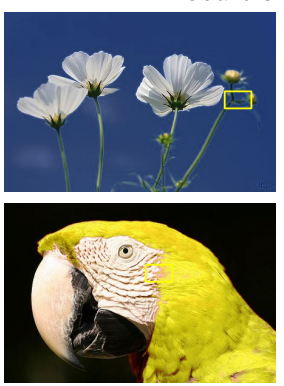
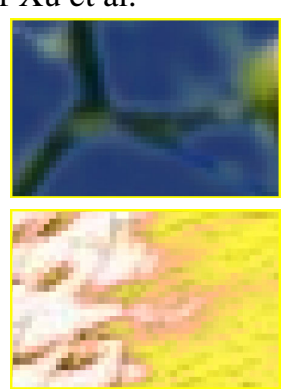
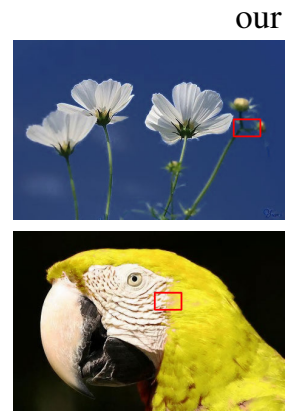

our result

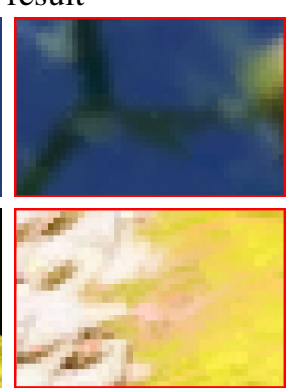

Figure 6: Results generated by Xu et al. [1] and by our method. The first column give the original images; the second and third columns are results generated by Xu et al.; the fourth and fifth columns are results generated by our method.

528 [32] Pellacini F, Lawrence J. Appwand: editing measured materials us- 564 2007;26(3):54-54

33] Xiao C, Nie Y, Tang F. Efficient edit propagation using hierarchical data structure. IEEE Transactions on Visualization and Computer Graphics 2011;17:1135-1147.

34] Bie X, Huang H, Wang W. Real time edit propagation by efficient sampling. Computer Graphics Forum 2011;30(7):2041-2048.

[35] Dong Y, Wang J, Tong X, Snyder J, Lan Y, Ben-Ezra M, et al. Manifold bootstrapping for svbrdf capture. ACM Transactions on Graphics 2010;29:98:1-98:10.

36] Lan Y, Dong Y, Wang J, Tong X, Guo B. Condenser-based instant reflectometry. Computer Graphics Forum 2010;29:2091-2098.

37] Ren P, Wang J, Snyder J, Tong X, Guo B. Pocket reflectometry. ACM Transactions on Graphics 2011;30:45:1-45:10.

38] Dong Y, Tong X, Pellacini F, Guo B. Appgen: Interactive material modeling from a single image. ACM Transactions on Graphics 2011;30(6):146:1-146:10.

39] Dana KJ, van Ginneken B, Nayar SK, Koenderink JJ. Reflectance and texture of real-world surfaces. ACM Transactions on Graphics 1999;18(1):1-34.

[40] Xu K, Wang J, Tong X, Hu SM, Guo B. Edit propagation on bidirectional texture functions. Computer Graphics Forum 2009;28(7):1871-1877.

41] Farbman Z, Fattal R, Lischinski D. Diffusion maps for edge-aware image editing. ACM Transactions on Graphics 2010;29:145:1-145:10.

42] Pharr M, Humphreys G. Physically Based Rendering: From Theory to Implementation. Morgan Kaufmann; 2004.

43] Akenine-möller T, Haines E, Hoffman N. Real-Time Rendering 3rd ed. AK peters; 2008.

44] Reeves WT, Salesin DH, Cook RL. Rendering antialiased shadows with depth maps. SIGGRAPH Computer Graphics 1987;21(4):283-291.

shadows with shadow maps. In: Computer Graphics International. 2001, p. 209-214.

46] Cant RJ, Shrubsole PA. Texture potential mip mapping, a new highquality texture antialiasing algorithm. ACM Transactions on Graphics
2000;19(3):164-184.

5 [47] Jon PE, Marcus DW, Martin W, Paul FL. Implementing an anisotropic texture filter. Computers and Graphics 2000;24(2).

[48] Pan M, Wang R, Chen W, Zhou K, Bao H. Fast, sub-pixel antialiased shadow maps. Computer Graphics Forum 2009;28:1927-1934.

569 [49] Van-hateren JH, Vander-schaaf A. Independent component filters of natural images compared with simple cells in primary visual cortex. Proceedings of the Royal Society 1998;B(265):359-366.

2 [50] Hyvärinen A, Hurri J, Hoyer PO. Natural image statistics: A probabilistic approach to early computational vision. Springer; 2009.

574 [51] Reshetov A. Morphological antialiasing. In: Proceedings of the Conference on High Performance Graphics 2009. HPG '09; 2009, p. 109-116.

576 [52] Zhang SH, Chen T, Zhang YF, Hu SM, Martin RR. Vectorizing cartoon animations. Visualization and Computer Graphics 2009;15(4):618-629.

578 [53] Lai YK, Hu SM, Martin RR. Automatic and topology-preserving gradient mesh generation for image vectorization. ACM Transactions on Graphics 2009;28(3):85:1-85:8

1 [54] Johannes K, Dani L. Depixelizing pixel art. ACM Transactions on Graphics 2011;30:99:1-99:8.

[55] Ma LQ, Xu K. Antialiasing recovery for edit propagation. In: Proceedings of the 10th International Conference on Virtual Reality Continuum and Its Applications in Industry. VRCAI '11; 2011, p. 125-130.

[56] Chen T, Cheng MM, Tan P, Shamir A, Hu SM. Sketch2photo: internet image montage. ACM Transactions on Graphics 2009;28:124:1-124:10.

8 [57] Ding M, Tong RF. Content-aware copying and pasting in images. The Visual Computer 2010;26:721-729.

590 [58] Huang H, Zhang L, Zhang HC. Arcimboldo-like collage using internet images. ACM Transactions on Graphics 2011;30(6):155:1-155:8.

592 [59] Du H, Jin X. Object cloning using constrained mean value interpolation. The Visual Computer 2012;

594 [60] Huang H, Fu T, Li CF. Painterly rendering with content-dependent natural paint strokes. The Visual Computer 2011;27(9):861-71. 


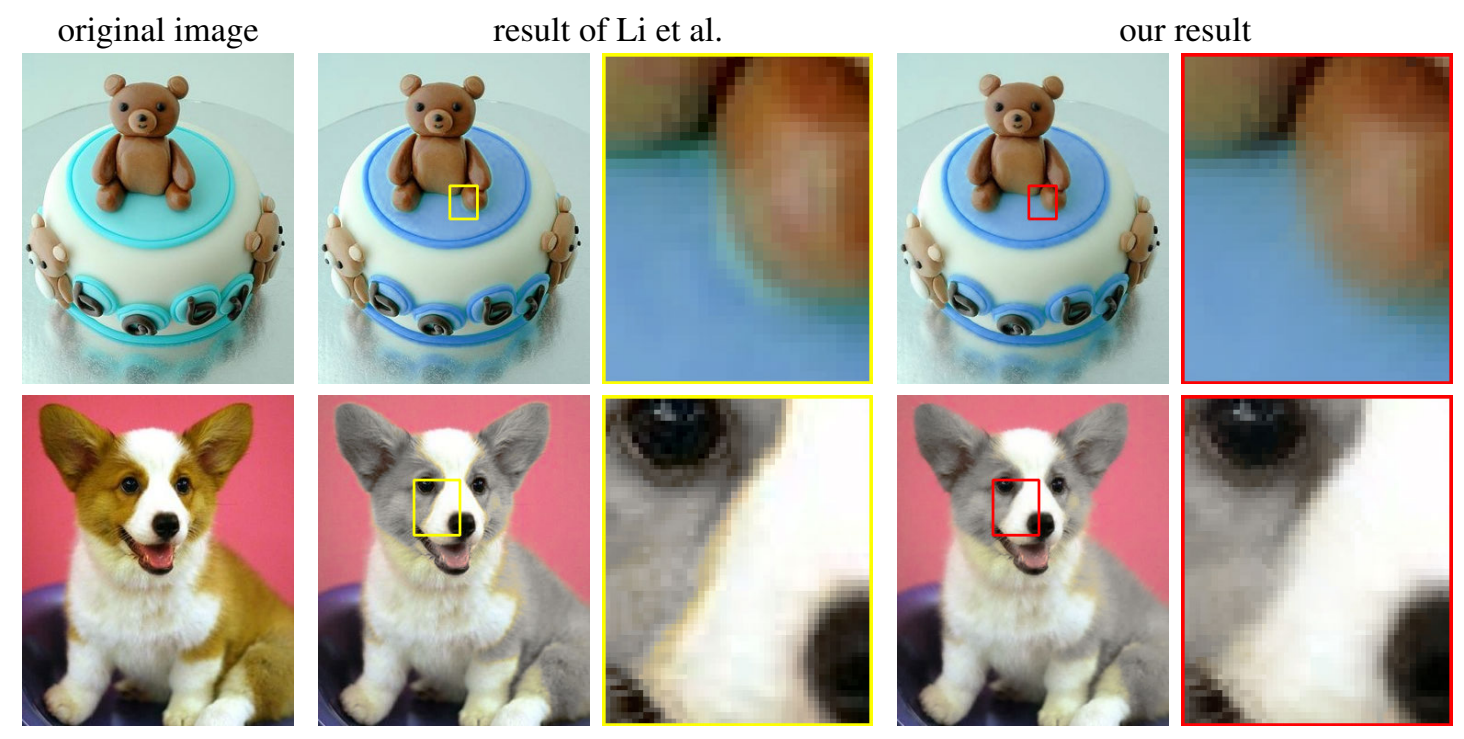

Figure 7: Results generated by Li et al. [2] and by our method. The first column give the original images; the second and third columns are results generated by Li et al.; the fourth and fifth columns are results generated by our method.
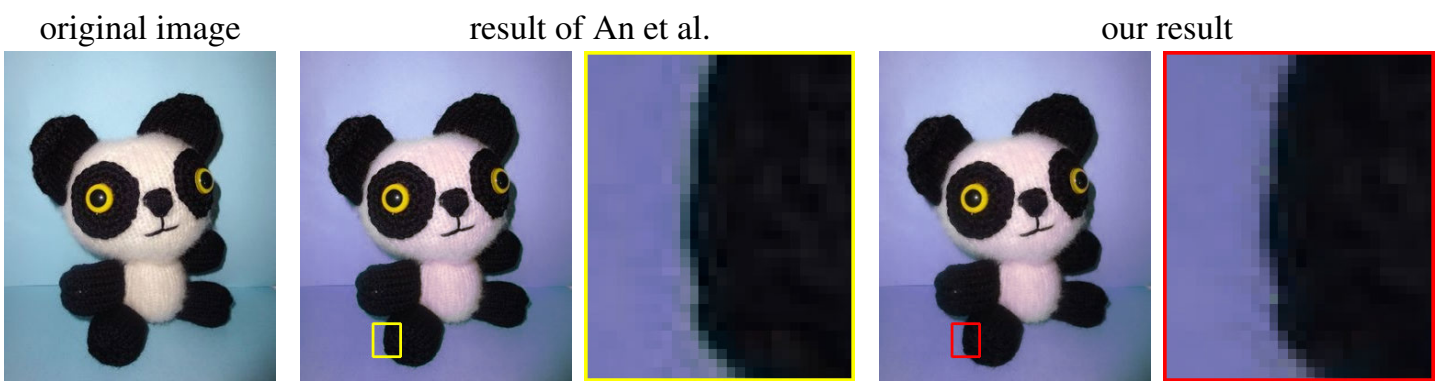

Figure 8: Results generated by An et al. [3] and by our method. The first column give the original images; the second and third columns are results generated by An et al.; the fourth and fifth columns are results generated by our method.

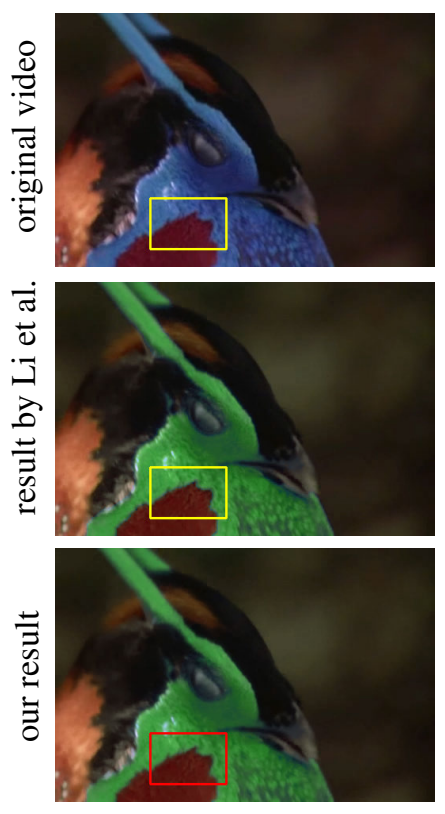

frame 237
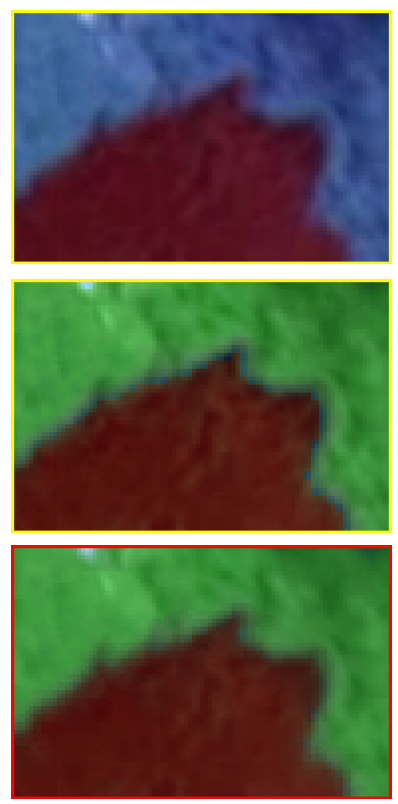

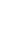
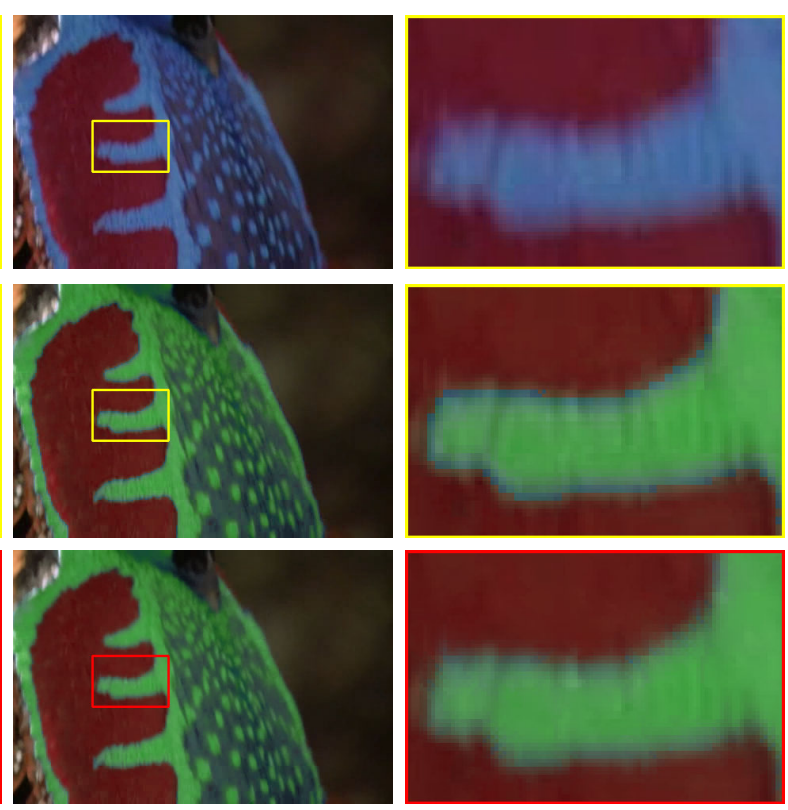

frame 259

Figure 9: Video results generated by Li et al. and our method. We have shown two frames of the video and clearly our method improves a lot along the boundaries. 